\section{ВЫДЕЛЕНИЕ ИНДИВИДУАЛЬНЫХ ПРИЗНАКОВ НА ЦИФРОВЫХ ИЗОБРАЖЕНИЯХ СЛЕДОВ БОЙКОВ}

\section{В. А. Федоренко}

кандидат физико-математических наук, доцент, заведующий лабораторией микроанализа и моделирования результатов применения оружия, Образовательно-научный институт наноструктур и биосистем Саратовского государственного университета E-mail: fed77@yandex.ru

\section{М. В. Корнилов}

программист лаборатории микроанализа

и моделирования результатов применения оружия,

Образовательно-научный институт наноструктур и биосистем

Саратовского государственного университета

E-mail: kornilovmv@gmail.com

Введение. Актуальность работы обусловлена широким внедрением автоматизированных баллистических идентификационных систем (АБИС) в баллистические лаборатории экспертных учреждений России. Баллистические системы позволяют автоматизировать проведение проверок по гильзотекам, содержащим тысячи однотипных объектов. Однако в отдельных случаях системы допускают «промахи», т.е. не могут найти в массиве электронной гильзотеки «парный» след (след, оставленный тем же экземпляром оружия, что и исследуемый). Кроме этого, иногда «парный» след из тестового массива ставится в конце приоритетного списка, что осложняет работу эксперта. Это обусловлено, в первую очередь, большим морфологическим разнообразием и высокой вариативностью индивидуальных признаков оружия, отобразившихся в следах бойков, а также неравномерным освещением следов из-за их сложной формы. Теоретический анализ. Исследования показали, что неравномерность яркости цифровых изображений следов бойков может быть сглажена путем применения метода гомоморфной обработки изображений. Анализ морфологии индивидуальных признаков оружия, отобразившихся в следах бойков более 30 моделей оружия, позволил выделить 6 основных морфологических типов признаков. Экспериментальное исследование. Разработаны эффективные алгоритмы выделения и бинаризации признаков в виде крупных пятен неопределенной формы на основе применения фильтра Винера и метода Ниблэка. Для выделения признаков в виде окружностей предложен метод, основанный на применении фильтра Канни. Данные алгоритмы могут найти применение при разработке программного обеспечения баллистических систем, а также при обработке цифровых изображений следов бойков при проведении экспертных исследований. Выводы. Метод гомоморфной обработки цифровых изображений может быть рекомендован для предварительной обработки исходных изображений. Впервые предложена классификация морфологических типов индивидуальных признаков. Разработаны алгоритмы бинаризации изображений с индивидуальными признаками в виде областей неопределенной формы и в виде окружностей.

Ключевые слова: следы бойков, гильзотека, обработка цифровых изображений, идентификация оружия, автоматизированные идентификационные баллистические системы.

\section{Введение}

В настоящее время в баллистических лабораториях экспертных учреждений достаточно широко используются автоматизированные баллистические идентификационные системы (АБИС). Они
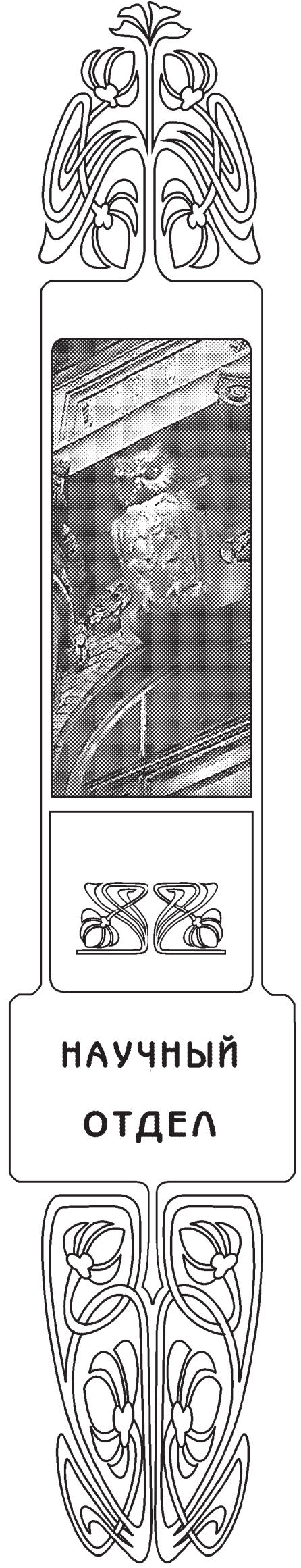
способны существенно облегчить работу эксперта, а в некоторых случаях позволяют решить задачи, которые не могут быть реализованы традиционным способом. Например, проведение идентификационных исследований по гильзотекам, содержащим тысячи однотипных объектов. Однако индивидуальные признаки оружия, отобразившиеся в следах бойков, имеют большое морфологическое разнообразие и высокую вариативность, а сами изображения - неравномерную яркость. Эти факторы серьезно усложняют процесс сравнения цифровых изображений следов бойков в автоматическом режиме баллистическими идентификационными системами. Предварительные исследования показали, что невозможно разработать единый уникальный алгоритм, который бы позволил одинаково эффективно выделять индивидуальные признаки с различной морфологией. Поэтому в работе было решено последовательно две задачи: 1 - впервые проведена классификация индивидуальных признаков следов бойка; 2 - для индивидуальных признаков часто встречающихся морфологических типов разработаны эффективные алгоритмы их выделения и перевода изображений, представленных в градациях серого, в бинарный вид. Корректность выделения индивидуальных признаков и бинаризация изображений во многом предопределяет эффективность последующего сравнения следов.

Бинаризация позволяет, с одной стороны, на порядок уменьшить объемы сравниваемых изображений без значимых для последующей идентификации искажений индивидуальных признаков, с другой - представить индивидуальные признаки в виде одноцветных простых фигур, более удобных для последующего их сравнения с аналогичными признаками бинарных изображений тестового массива.

\section{Теоретический анализ}

Абсолютное большинство изображений следов бойков имеет неравномерную яркость.
Этот фактор серьезно затрудняет единообразие выделения индивидуальных признаков не только на разных изображениях, но и на разных участках одного изображения. Исследования показали, что наиболее простым и эффективным методом выравнивания освещения монохромных цифровых изображений является метод гомоморфной обработки [1]. Суть данной методики заключается в следующем. Считается, что неравномерность освещения, обусловленная формой бойка, изменяется медленнее, чем неравномерность освещения, обусловленная микрорельефом следа. Поэтому низкочастотная (НЧ) составляющая изображения обусловлена, в первую очередь, неравномерностью освещения следа бойка. Высокочастотная (ВЧ) составляющая изображения сформирована локальными неоднородностями микрорельефа. Таким образом, исходное изображение можно представить в виде перемножения двух изображений - низкочастотного и высокочастотного. Чтобы ослабить неравномерность освещения, необходимо выделить ВЧ составляющую изображения, затем ее сложить с исходным, но с разными коэффициентами. Исходное изображение следует умножить на понижающий коэффициент, а изображение ВЧ составляющей на повышающий.

Для получения низкочастотной составляющей исходного изображения размером $250 \times 250$ пикселей применялся двухмерный гауссовский фильтр размерностью $25 \times 25$. Расчеты, проведенные в соответствии с методикой гомоморфной обработки изображений, показали хорошие результаты. Иллюстрация действия данной методики представлена на рис. 1. Видно, что после выравнивания освещения стал хорошо различим признак в виде длинной темной линии в правом верхнем углу (выделен стрелкой на рис. 1, 2 ). Полученное изображение лучше исходного и является более однородным по яркости. Исходные изображения получены на АБИС «PISC».

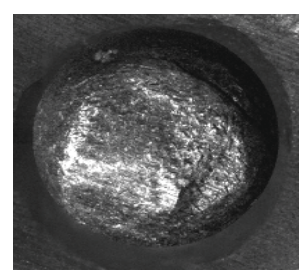

$a$

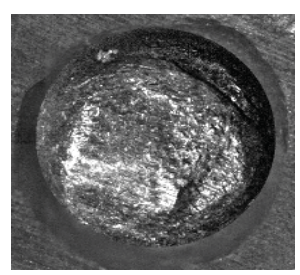

б

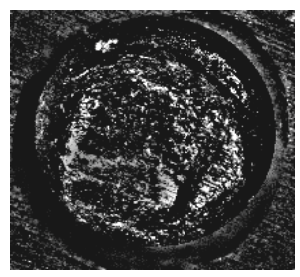

B

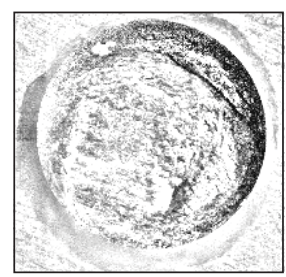

2

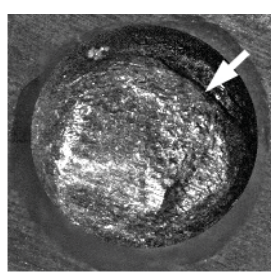

$\partial$

Рис. 1. Изображение следа бойка АКМ: $a$ - исходное изображение следа бойка; $\sigma$ - изображение после действия низкочастотного гауссовского фильтра; в - изображение после высокочастотной фильтрации (потеря НЧ информации); г - изображение после взятия экспоненты ВЧ составляющей изображения; $\partial$ - изображение с выровненной яркостью 
После отработки методики выравнивания яркости изображений была проведена классификация основных форм (типов) индивидуальных признаков оружия, отобразившихся в следах бойка. В процессе анализа следов бойков более 30 моделей оружия удалось выделить 6 основных морфологических типов индивидуальных признаков (рис. 2) (без учета групповых признаков, таких как форма, диаметр, глубина следа и т.д.), на основе которых могут быть сформированы еще и дополнительные комбинированные типы:

1) признаки в виде крупных неоднородностей микрорельефа неопределенной формы (рис. 2, a);

2) признаки в виде относительно мелких топографических неоднородностей микрорельефа (рис. 2, б);

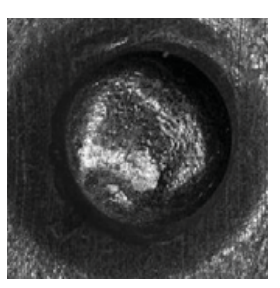

$a$

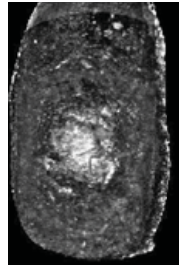

6

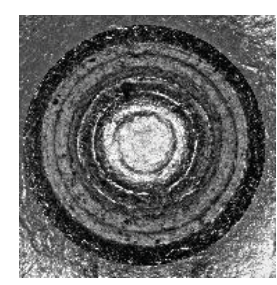

B

3) признаки в виде вложенных друг в друга окружностей (рис. 2, в);

4) признаки в виде трасс, расположенных в динамической части следов бойка (рис. 2,2 );

5) признаки в виде наслоений (рис. 2, 2 ), данный тип признаков характерен для изображений, полученных на растровом электронном микроскопе;

6) признаки в виде контуров или топографических неоднородностей с четко выделенными границами (рис. $2, e$ ), данный тип признаков характерен для изображений, полученных на растровом электронном микроскопе;

7) комбинированные типы (комбинации основных типов, обычно не более двух). Например, на изображении кроме признаков в виде окружностей могут присутствовать еще и отдельные признаки в виде пятен неопределенной формы.

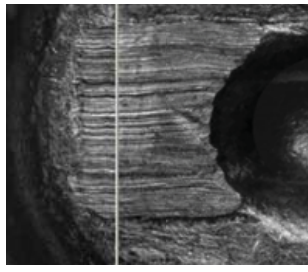

2

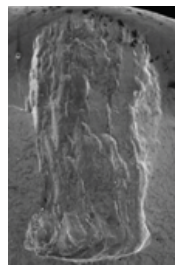

$\partial$

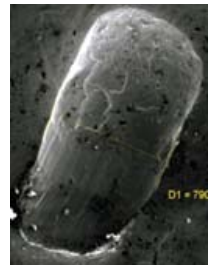

$e$

Рис. 2. Основные морфологические типы индивидуальных признаков

Одним из основных негативных факторов, отрицательно влияющих на сравнение признаков, является их вариативность. Причины вариативности признаков обычно связывают с технологическими допусками на производство оружия и патронов, уникальностью каждого выстрела. Однако достаточно интересной является еще одна причина, которая может внести существенный вклад в вариативность признаков - это наличие шероховатости на поверхности капсюля еще до нанесения по нему удара бойком. В процессе выполнения работы были изучены основные виды неоднородностей поверхности капсюлей. На рис. 3 показаны изображения основных типов рельефов поверхности капсюлей, полученные с помощью баллистической системы «POISC». Области высокой фокусировки представлены в виде окружностей диаметром, равным среднему значению диаметра следа бойка - 1,5 мм. В результате жесткого удара закаленным бойком по поверхности капсюля плавные и небольшие по высоте неоднородности сглаживаются. Удар бойка в какой-то степени аналогичен применению сглаживающего фильтра, размывающего мелкие неоднородности. Однако ярко выраженные неоднородности поверхности сохраняются и складываются с микрорельефом отпечатка бойка. Это приводит к некоторым искажениям признаков.

Характер неоднородностей поверхности капсюлей (рис. 3) следует учитывать на этапе предварительной обработки изображений. Например, при выделении индивидуальных признаков в виде крупных областей изменения яркости изображения, вызванные шероховатостью поверхности капсюлей, удается значительно ослабить путем применения сглаживающих пространственных фильтров, таких как фильтры Гаусса, Винера, медианный фильтр и др. Маска (ядро) фильтра должна быть меньше размерных характеристик индивидуальных признаков, но больше неоднородностей поверхности капсюля. Например, для крупноразмерных признаков это условие легко обеспечить путем применения сглаживающих фильтров с размерами не менее чем $9 \times 9$ элементов.

Однако эта проблема остается пока не решенной для изображений с индивидуальными признаками в виде небольших пятен, соизмеримых с естественными шероховатостями поверхности капсюля. Возможно, анализ морфологии и структуры неоднородностей на поверхности капсюля за пределами следа бойка позволит применить некоторые статистические методы для их сглаживания уже на изображении самого следа. 


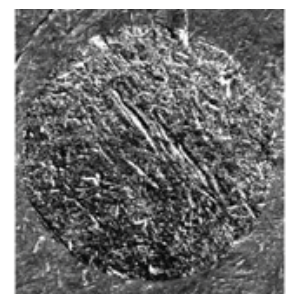

$a$

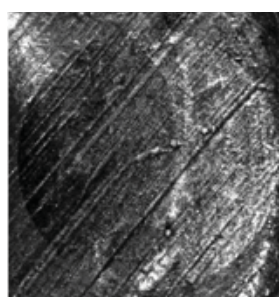

6

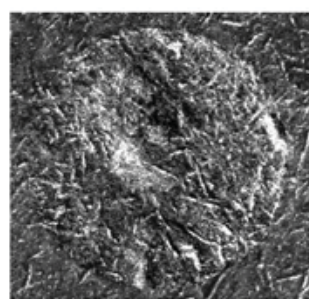

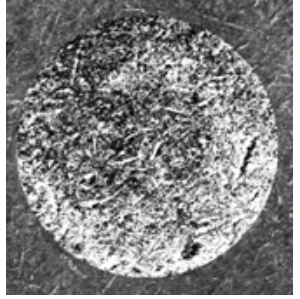

2

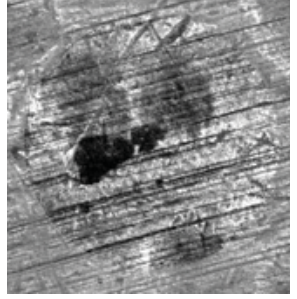

$\partial$

Рис. 3. Изображения неоднородностей поверхности капсюлей: $a$-неоднородности в виде хаотично расположенных мелких впадин и бугорков с присутствием нескольких протяженных узких борозд; $\sigma$ - неоднородности в виде ярко выраженных трасс от валков, оставленных в процессе прокатки фольги для капсюлей; 8 - крупная вмятина на фоне хаотично расположенных микронеоднородностей; 2 - мелкие шероховатости; $\partial$ - пятна неопределенной формы от окисления поверхности металла и остатков защитного лака (темное пятно) на фоне трасс от валков

\section{Экспериментальное исследование}

После выравнивания освещения и некоторого размытия изображений с целью сглаживания пространственных шумов следует один из основных этапов обработки изображения - перевод изображения, представленного в градациях серого, в двухцветное, в данном случае в черное и белое. В идеале признаки необходимо выделить одним цветом, например белым, а фон и размытые шумы - другим, например черным. Однако восприятие яркостных переходов человеком и компьютером разное. Глаз человека в определенных случаях воспринимает области, имеющие одну яркость, как принципиально разные по яркости (например, оптические иллюзии), а компьютер считывает их как пятна с одной яркостью. Чтобы компьютер выделял признаки аналогично человеку, очевидно, необходимо оперировать адаптивным порогом бинаризации. То есть порог бинаризации для каждого пикселя (точки) должен определяться с учетом яркости окружающих пикселей.

Исследования, проведенные с цифровыми изображениями следов с признаками в виде областей неопределенной формы и признаками в виде окружностей, показали следующее. Одними из наиболее простых и эффективных алгоритмов выделения признаков первого типа (в виде крупных пятен неопределенной формы) оказались алгоритмы, основанные на применении фильтра Винера и метода Ниблэка. Для первого случая может быть рекомендована следующая последовательность фильтраций исходного изображения: выравнивание освещения, размытие Гауссианом, сглаживание фильтром Винера, бинаризация, морфологическое размыкание-замыкание. Применение фильтра Винера приводит к размытию темных участков и сохранению контрастных границ. Пример реализации данного алгоритма представлен на рис. $4 a, \sigma, в$.

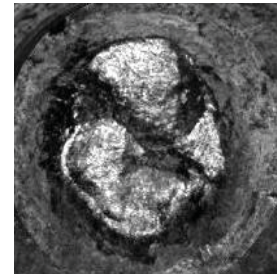

$a$

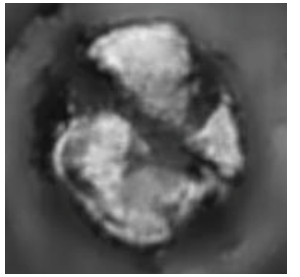

$\sigma$

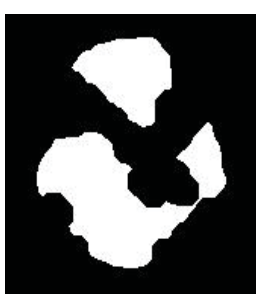

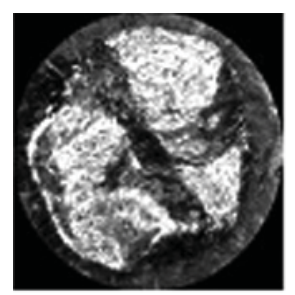

2

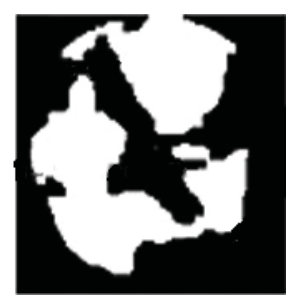

$\partial$

Рис. 4. Бинаризация с применением фильтра Винера: $a$ - исходное изображение; $\sigma$ - результат применения фильтра Винера; в - бинарное изображение; 2 - исходное изображение следа бойка; $\partial$ - изображение, представленное на рис. 4 , г, бинаризованное методом Ниблэка

По сравнению с предыдущим методом бинаризации, адаптивная бинаризация методом Ниблэка позволяет достичь более высокой скорости обработки изображений. Метод используется на практике для быстрой фильтрации достаточно контрастных изображений, на которых отсутствуют сильно зашумленные области с плавны- ми переходами яркости. Выравнивание яркости изображений методом гомоморфной обработки не приводит к существенному уменьшению перепадов яркости на границах областей-признаков. Поэтому при применении метода Ниблэка гомоморфная обработка изображений может быть сохранена, хотя и не является обязательной. 
Кроме этого, метод Ниблэка имеет высокую устойчивость работы в широком динамическом диапазоне яркостей. Например, расчеты показали, что результаты бинаризации одних и тех же изображений с яркостью, сдвинутой на 60 единиц (из возможных 256), отличаются менее чем на 5\% по таким дескрипторам, как площадь (S) и периметр (P).

Принцип действия метода Ниблэка состоит в варьировании порога бинаризации $B$ по яркости от точки к точке на основании локального значения стандартного отклонения. Порог яркости в точке $(x, y)$ рассчитывается по формуле

$$
B(x, y)=\mu(x, y)+k \sigma(x, y),
$$

где $\mu(x, y), \sigma(x, y)$ - среднее и стандартное отклонение выборки для некоторой окрестности точки.

Размер окрестности (маски) выбирается таким, чтобы сохранить на изображении индивидуальные признаки, но при этом понизить влияние пространственных неоднородностей поверхности капсюля. Для изображений следов бойков размером $250 \times 250$ радиус окрестности составляет порядка 50 пикселей. Значение $k$ определяет, какую часть границы признака (объекта) взять в качестве самого признака. Значение $k=+0,2$ задает достаточно хорошее разделение объектов, когда они представлены белым цве- том. На рис. 4, г, д представлены исходное изображение и результат его бинаризации методом Ниблэка. Видно, что основные характеристики индивидуальных признаков в виде пятен неопределенной формы сохранены.

Из рис. 4 видно, что данные алгоритмы могут быть успешно применены для выделения индивидуальных признаков, представляющих собой области неопределенной формы, размерные характеристики которых намного больше соответствующих характеристик шероховатостей поверхности капсюля.

Бинаризация изображений следов бойков с признаками в виде окружностей с применением рассмотренных выше методов приводит практически к полной их потере. Для бинаризации таких изображений эффективным является следующий алгоритм: выравнивание освещения; фильтр Гаусса (легкое размытие для удаления точечных шумов); контрастно повышающий фильтр; фильтр Канни для выделения границ областей в виде тонких линий; морфологическое размыкание-замыкание для утолщения линий и удаления коротких [2]. Из представленных на рис. 5 изображений видно хорошее совмещение признаков, выделенных в виде тонких колец. Данный алгоритм может быть рекомендован для бинаризации изображений с признаками в виде окружностей.

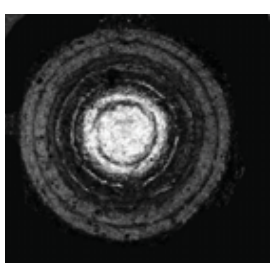

$a$

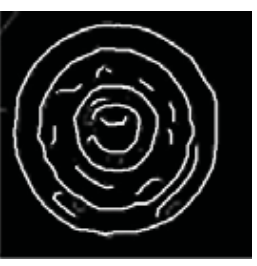

6

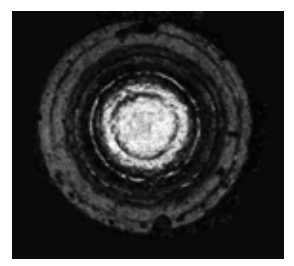

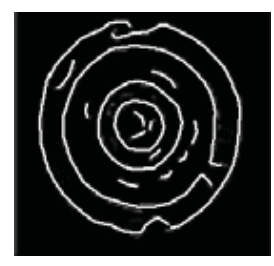

2

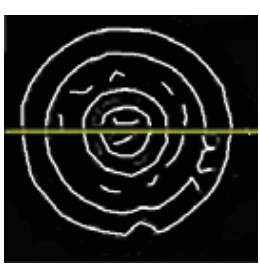

$\partial$

Рис. 5. Следы бойка с признаками в виде окружностей: $a$ - исходное изображение следа бойка № $1 ; \sigma-$ бинарное изображение следа бойка № $1 ;$ в - исходное изображение следа бойка № $2 ; 2$ - бинарное изображение следа бойка № $2 ; \partial-$ совмещение признаков на бинарных изображениях следов бойков № 1 и № 2

Проведенные исследования показали, что классификация изображений следов бойков по морфологическому типу индивидуальных признаков позволяет разработать для каждого независимого типа признаков эффективные алгоритмы их выделения и бинаризации. Представленные алгоритмы позволяют эффективно выделять индивидуальные признаки в виде пятен неопределенной формы и в виде окружностей. Впервые инициирован вопрос о влиянии шероховатостей поверхности капсюлей на вариативность индивидуальных признаков оружия, отобразившихся в следах бойков.

\section{Выводы}

Показано, что метод гомоморфной обработки цифровых изображений позволяет сгладить неоднородность по яркости исходных изображений следов бойков.

Разработана классификация морфологических типов индивидуальных признаков.

Разработаны алгоритмы выделения признаков в виде крупных областей и в виде окружностей и последующей бинаризации изображений следов бойков.

Выделен новый механизм вариативности индивидуальных признаков - наличие шероховатостей на поверхности капсюля. 


\section{Список литературы}

1. Фисенко Т. Ю., Фисенко В. Т. Исследование и разработка методов улучшения подводных изображений / Филиал ОАО «Корпорация "Комета" - научно-проектный центр оптоэлектронных комплексов наблю- дения». Санкт-Петербург. 2011. С. 294-298. URL: http://www.oop-ros.org/maket2012/part7/7.18.pdf (дата обращения: 10.03.2014).

2. Гансалес Р., Вудс Р. Мир цифровой обработки. Цифровая обработка изображений : пер. с англ. / под ред. П. А. Чочиа. М. : Техносфера, 2005. 1072 с.

\title{
Allocation of Individual Attributes on Digital Images of the Firing Pin Traces
}

\author{
V. A. Fedorenko \\ Education Research Institute of Nanostructures and Biosystems \\ of the Saratov State University, \\ 83, Astrakhanskaya, Saratov, 410012, Russia \\ E-mail: fed77@yandex.ru

\section{V. Kornilov} \\ Education Research Institute of Nanostructures and Biosystems \\ of the Saratov State University, \\ 83, Astrakhanskaya, Saratov, 410012, Russia \\ E-mail: kornilovmv@gmail.ru
}

Introduction. Automated ballistic identification systems allow automation of inspections by array firing pin traces containing thousands of similar objects. However, sometimes the system allow «mistakes», ie can not find 'doubles' trail in database. In addition, quite often «doubles» trail of test array is placed on the priority list is far from its beginning. This is due, primarily, large morphological variety and high variability of individual attributes of weapons displayed in the firing pin tracks, as well as uneven lighting traces due to their complex forms. Theoretical analysis. Studies have shown that non-uniformity of brightness of digital images traces of the firing pin can be smoothed by applying the homomorphic image processing method. Analysis of the morphology of individual signs weapons displayed in the tracks of the strikers 30 models of weapons, allowed to identify six main morphological types of individual attributes. Experimental investigation. Efficient algorithms for feature extraction of the form of large irregularly shaped spots by applying the Wiener filter and method Niblek developed. For feature extraction in the form of circles, we propose a method based on the use of the Canny filter. Conclutions. Studies have shown: homomorphic method of digital image processing can be recommended for pre-processing raw images; classification of morphological types of individual signs developed; algorithms binarization images features in the form of large irregularly shaped areas and in the form of circles developed.

Key words: traces of the firing pin, cartridge cases database, digital imaging, identification of weapons, automated ballistic identification system.

\section{References}

1. Fisenko T. Yu., Fisenko V. T. Issledovanie i razrabotka metodov uluchsheniya podvodnych isobrazheniy (Research and development of methods of improvement of underwater images). Filial OAO «Korporatsiya "Kometa" - nauchno-proektniy centr optoelectronnich kompleksov nabludeniya» [Branch of OJSC «Corporation "Comet" - research and design center of opto- electronic surveillance complexes»], St.-Petersburg, 2011, pp. 294-298. Available at: http://www.oop-ros. org/maket2012/part7/7.18.pdf (accesses 10 March 2014).

2. Gonzales Rafael C., Woods Richard E. Digital Image Processing. 2nd ed., Prentice Hall, Upper Saddle River, N.J., 2002 (Russ. ed.: Gonsales R., Vuds R. Tsifrovaia obrabotka izobrazhenii. Moscow, Tekhnosfera Publ., 2005. 1072 p.). 